\title{
Le rapport aux TIC des cadres : réflexions sur l'usage de l'entretien biographique dans une perspective longitudinale
}

Executives' Relationship To Information and Communications Technology (ICT): The Use of Biographical Interviews in a Longitudinal Perspective

\section{Cindy Felio}

\section{OpenEdition}

Journals

Édition électronique

URL : http://journals.openedition.org/edc/6049

DOI : $10.4000 /$ edc.6049

ISSN : 2101-0366

Éditeur

Université Lille-3

Édition imprimée

Date de publication : 1 décembre 2014

Pagination : 145-164

ISBN : 978-2-917562-12-3

ISSN : $1270-6841$

\section{Référence électronique}

Cindy Felio, « Le rapport aux TIC des cadres : réflexions sur l'usage de l'entretien biographique dans une perspective longitudinale », Études de communication [En ligne], 43 | 2014, mis en ligne le 01 décembre 2014, consulté le 16 mai 2019. URL : http://journals.openedition.org/edc/6049; DOI : 10.4000/edc. 6049 
Varias... 



\section{CINDY FELIO 1 \\ LE RAPPORT AUX TIC DES CADRES : \\ RÉFLEXIONS SUR L'USAGE DE L'ENTRETIEN \\ BIOGRAPHIQUE DANS UNE PERSPECTIVE \\ LONGITUDINALE}

L'introduction des technologies de l'information et de la communication (TIC) dans les organisations s'est accompagnée, dès le début de leur intégration dans les procès de travail, d'une reconfiguration des activités professionnelles. Bien sûr, ces dispositifs communicationnels ne jouent pas un rôle strictement déterminant dans cette transformation de l'organisation du travail. Il s'agirait plutôt d'une détermination réciproque entre, d'une part, l'intégration de ces dispositifs et, d'autre part, l'organisation (Burnier, 2012a); des effets mutuels qui convoquent alors de nouvelles modalités de réalisation des activités (Brousseau et Rallet, 1997). À l'issue de ce processus, l'expérience du travail endosse une nouvelle facette : celle de sa médiatisation ${ }^{2}$. Cette forme renouvelée des organisations

\footnotetext{
$1 \quad$ Lauréate du 2e prix « jeunes chercheurs » 2014 de la SFSIC.

2 Traditionnellement en Sciences de l'Information et de la Communication, le terme de «médiatisation » renvoie aux médias de masse. Dans le contexte de notre étude, nous l'employons pour caractériser la reconfiguration des activités de travail du fait de l'introduction des TIC en milieu professionnel. Ces dispositifs étant définis, non pas seulement comme de simples intermédiaires mais comme des médias (considérant, à l'instar de Yves Jeanneret -2009-, que leur usage est créateur de social), le terme de «médiatisation » nous semble pertinent pour saisir la reconfiguration technique de l'activité professionnelle.
} 
susciterait la mobilisation subjective et cognitive de ses acteurs et ferait appel à plus d'autonomie ${ }^{3}$. Les principales caractéristiques du travail médiatisé par les TIC s'articuleraient autour de la mutation du rapport au temps ${ }^{4}$ (temps réel, accélération), de l'exploitation des capacités attentionnelles ${ }^{5}$ (disruption, attraction cognitive), et de la traçabilité 6 (contrôle, reporting).

La catégorie socioprofessionnelle des cadres semble la plus concernée par la digitalisation des activités de travail, eu égard à leur taux d'équipement en TIC professionnelles, notamment dans leur version mobile (Kalika et Romelaer, 2006). Depuis les années 1980 (et avec une recrudescence dans les années 1990), la majorité de leurs activités communicationnelles est médiatisée par la technique. La littérature en sciences humaines et sociales, fortement documentée sur cette catégorie de salariés, met en évidence toute la complexité à appréhender un quotidien de travail de plus en plus instrumenté, fragmenté et dont la temporalité revêt une dimension de porosité. Les travaux portant sur les usages numériques des cadres (BobillierChaumon, 2012; Sarnin, 2012) mettent en évidence un rapport ambivalent, conjuguant des dimensions de contrôle, d'efficacité et d'autonomie, avec des facettes relevant de la perte de sens du travail, de la dissolution des collectifs et de la dépendance à l'égard de la technique. Soulignons, par ailleurs, que la plainte de l'hyperconnexion des cadres avec le travail est fortement présente dans les médias de masse ${ }^{7}$, alors que le discours organisationnel sur cette question demeure absent ${ }^{8}$. La prise en compte du discours hégémonique portant sur la figure du cadre contemporain (Bouilloud, 2012) permettrait de répondre à l'indicibilité de cette plainte dans les organisations : puisque la représentation sociale du «bon cadre »

\footnotetext{
3 Voir Burnier (2012b), Walkowiak et Greenan (2005), Brynjolfsson et Hitt (2000).

$4 \quad$ Voir Menga (2012), Rosa (2010), Carayol (2005).

5 Voir Broadbent (2012), Datchary et Licoppe (2007), Gonzàlez et Mark (2004).

6 Voir Chevallet et Moatty (2012), Lépine (2001), Cochoy et De Terssac (2000).

7 Le titre du périodique L'Express datant de mai 2012 en témoigne : «Les cadres ne veulent plus être branchés en permanence ».

8 Plusieurs références et travaux de recherche donne à penser cette carence de discussion autour des usages des TIC au sein des organisations: Felio (2014), Boudokhane-Lima et Carayol (2014), Lafranchise (2013), Document ANR $(2009,12)$.
} 
prône l'engagement professionnel et affectif envers l'entreprise, ainsi que le surinvestissement au travail, l'intégration et la diffusion des valeurs (Vannereau, 2012 ; Le Goff, 1995), la plainte ne constitueraitelle pas une menace, pour les cadres, de «perdre la face» au sens goffmanien?

Afin de mieux comprendre cette relation ambiguë que semblent entretenir les cadres avec les dispositifs, une étude qualitative longitudinale a été réalisée auprès d'un échantillon de 62 cadres 9 $\left(\right.$ Felio, 2013) ${ }^{10}$. L'un des objectifs principaux consistait à mettre en lumière le vécu de cadres-usagers non seulement à partir du rôle actif qu'ils investissent dans l'appropriation des artefacts communicationnels, mais également de leurs capacités réflexives en tant qu'individus éprouvant la médiatisation de leur activité au quotidien. Cet article se propose d'étayer les potentialités offertes par une méthodologie d'étude des usages articulant conjointement une approche biographique et une dimension longitudinale. Nous développons, dans le corps de ce papier, aussi bien les caractéristiques de la temporalité expérimentale de l'étude ${ }^{11}$ (évolution des usages des TIC), que le vécu des sujets investis dans une recherche doctorale portant sur leurs propres pratiques communicationnelles. La réflexivité des cadres de l'étude, comme la posture du chercheur dans une approche à la fois biographique et diachronique, sont ici mises à l'honneur.

\section{Biographie et temporalité au cour du design de la recherche}

\section{Cadre conceptuel}

Pour appréhender les pratiques communicationnelles des cadres et leurs enjeux psychosociaux (soit l'objet de la recherche doctorale sur laquelle repose le contenu du présent article), un métissage conceptuel a été privilégié, conjuguant les Sciences de l'Information et de la Communication et la Psychologie du travail. En SIC, l'approche de la médiation développée par Yves Jeanneret (op. cit.), nous invite à saisir les dispositifs en leur qualité de médias, nous

9 Échantillon composé de 62 cadres exerçant leurs fonctions dans la région Aquitaine.

10 Thèse associée au programme de recherche international DEVOTIC.

$11 \mathrm{~T}=1$ an. 
permettant ainsi d'analyser de manière complexe le rôle des TIC dans les facteurs de risques psychosociaux au travail ${ }^{12}$. En réfutant toute logique déterministe des pratiques communicationnelles vis-à-vis de l'exposition aux risques, cette perspective consiste à éviter l'écueil d'une logique binaire opposant l'individu et les objets techniques, en raisonnant au prisme d'un modèle triangulaire « individu-technique-organisation» (Bernard, 2000). En mobilisant la perspective processuelle de la communication (Cherba et Vásquez, 2012; Cordelier et al., 2011), nous accordons un intérêt fort au processus de construction du sens des pratiques, notamment en prenant en compte les discours interprétatifs des acteurs sur les situations qu'ils vivent ${ }^{13}$. Une telle approche apparaît, selon Jean-Luc Bouillon (2003), particulièrement appropriée pour les études d'usage des TIC et les mutations organisationnelles associées. Conjointement à ce regard communicationnel, nous convoquons l'approche de la psychodynamique du travail (instituée par Christophe Dejours) pour comprendre la «résonance psychosociale (Amado, 2010) de l'expérience des TIC dans le quotidien des sujets. Ce regard clinique permet de mettre en exergue la mobilisation des individus et des collectifs à travers les stratégies ${ }^{14}$ élaborées pour pallier les

12 Dans le corps de cet article, nous employons le terme de «médiatisation » pour rendre compte de la reconfiguration des activités professionnelles du fait de l'intégration des TIC dans les procès de travail. Ce choix s'explique aussi par la volonté de ne pas assimiler la médiation technique à la définition commune de la notion de médiation qui renvoie à une posture de tiercéité visant la conciliation, la formation de compromis et de résolution de problème entre au moins deux individus (Barbot et Lancien, 2003). En suivant Yves Jeanneret (op. cit.), on pourrait dire que la notion de médiation renvoie à un processus qui « concerne l'opérativité symbolique [...] et sociale [...] des dispositifs », lesquels, en tant que médias, médiatisent notre expérience, à savoir, dans le cas présent, celle du travail.

13 Puisque cette approche offre l'opportunité d'interroger le processus de construction en situation, elle apparaît particulièrement pertinente pour répondre à nos objectifs de recherche, à savoir comprendre la manière de faire face aux incidents critiques passés (contribuant, en filigrane des ajustements individuels, à assurer le fonctionnement de l'organisation) mais appréhender le sens des pratiques révélées dans le cadre du dispositif méthodologique de l'étude (soit la dimension performative inhérente à la conduite d'entretiens biographiques).

14 Dans le champ de la psychodynamique du travail, les «stratégies » désignent à la fois la mobilisation consciente de ressources (intelligence pratique, créativité, équipement, environnement, organisation) et les défenses au sens psychanalytique du terme (visant principalement à se protéger contre la peur et à euphémiser la réalité pour mieux la supporter). 
contraintes inhérentes à l'organisation du travail (Molinier et Flottes, 2012 ; Dejours, 1988). Appréhender le caractère processuel du développement de ces stratégies permet de comprendre la dialectique plaisir/souffrance au travail : transposée à notre objet de recherche, la psychodynamique du travail met à notre disposition des outils pour distinguer les usages numériques contribuant à préserver la santé (dans une perspective dite "salutogène ») des usages dits «pathogènes », favorisant l'apparition de troubles psychosociaux.

$\mathrm{Au}$ cœur d'une épistémologie résolument compréhensive, cette transdisciplinarité, croisant les SIC et la Psychologie du travail, nous invite à saisir les pratiques communicationnelles d'un groupe social (en l'occurrence, ici, celui des cadres confrontés aux TIC) à partir du vécu expérientiel des individus qui le composent. Ainsi, la méthode biographique est apparue comme particulièrement appropriée dans le sens où elle consiste à recueillir l'explicitation de "récits thématiques relatifs à des moments vécus ou de récits se rapportant à des pratiques individuelles, souvent professionnelles »(Muchielli, 2009, 79).

\section{L'intérêt pour la réflexivité des sujets d'étude}

Comme le précise Patrice De La Broise (2008, 108), la démarche d'entretien biographique consiste en une :

situation dialogique dans laquelle la «mise en récit» relève nécessairement de la co-énonciation et par laquelle l'objet de questionnement est contextualisé dans une histoire qui le déborde et lui donne sens.

La dimension de réflexivité apparaît ici comme essentielle : l'exercice d'explicitation de ses propres pratiques communicationnelles engage le sujet au cœur d'un processus d'actualisation de soi. À travers un récit rétrospectif sur les pratiques et les évènements liés à l'usage professionnel des TIC que le sujet peut se remémorer, l'approche biographique entraine un exercice d'argumentation pour mettre en évidence ce qui fait sens dans son histoire. Partant, nous cherchons à recueillir des témoignages éprouvés et affectés reposant sur l'histoire des sujets, jalonnée d'anecdotes et d'événements significatifs en lien avec les usages des TIC. Par là même, nous souhaitons questionner les stratégies d'usage sous l'angle de la mobilisation rationnelle: 
les sujets élaborent-ils des comportements d'ajustement de manière consciente (Quéré, 1990)?

En concédant un intérêt pour les événements marquants dans le parcours des cadres-usagers, nous prenons en compte le bouleversement d'intelligibilité que leur expérience suscite (Bensa et Fassin, 2002). Parce que l'incident critique ${ }^{15}$ bouleverse le statu quo (Quéré, 2006), génère de l'incertitude et de l'instabilité, il joue le rôle de révélateur du sens des pratiques. Le vécu d'un événement constitue indéniablement une expérience de réflexivité et de construction du sens. Outre les incidents relatifs à des situations médiatisées par les TIC, la dimension longitudinale que nous introduisons dans l'étude permet également de questionner le parcours d'appropriation et de réappropriation ${ }^{16}$ des dispositifs dans une temporalité expérimentale définie. Comme le préconise Louis Quéré à propos de l'analyse d'un événement, c'est "ce pouvoir d'ouverture et de clôture, d'initiation et d'éclairage, de révélation et d'interpellation qu'il nous faut explorer» (ibid., 189).

Puisque les usages professionnels des TIC ne font pas l'objet de discussions au sein des organisations, nous considérons que l'exercice discursif et réflexif que nous initions auprès des sujets peut représenter, en lui-même, un «événement biographique » (Leclerc-Olive, 1997), ayant un impact sur leur manière de voir et de se comporter avec les dispositifs. Soulignons que l'objectif de la dimension longitudinale de la recherche est fondamentalement exploratoire : en effet, le but initial ne consiste pas à orienter un changement mais de questionner la possible existence d'une reconfiguration des pratiques. Il s'agit d'inclure dans l'étude une dimension réflexive en mettant à l'épreuve le contexte propre qu'engage le protocole de recherche sur le rapport

15 À partir d'une revue de la littérature portant sur le concept d'incident critique (initialement introduit par Flanagan en 1954), nous formulons la définition suivante : "Un incident critique est un évènement vécu de manière significative, chargé émotionnellement, à l'origine d'un changement de comportement et/ou d'attitude à l'égard d'un objet » (Felio, op. cit., 222).

16 Le vécu d'un événement contribuant à bouleverser la manière de voir et de se comporter à l'égard d'un objet, la trajectoire de l'usage des TIC peut apparaître vulnérable. Concevant le parcours d'appropriation des techniques de manière non-linéaire (à l'instar de Riccio et Commandre, 2009), nous nous intéressons à la mobilisation individuelle et subjective de l'usager pour comprendre l'incertitude générée par les incidents critiques et la reconstruction du sens des pratiques. C'est pourquoi évoquonsnous les possibilités de « réappropriation ». 
aux TIC qu'entretiennent les sujets. À l'instar de la pragmatique dialogique théorisée par Francis Jacques ${ }^{17}$, nous considérons que la relation interlocutive entre le chercheur et le sujet d'étude engage les deux protagonistes dans un rôle concomitant de producteurs de sens. Autrement dit, ils demeurent tous deux responsables du sémantisme des propos échangés. Le vécu de l'entretien biographique (comme tout dispositif de recherche impliquant la participation d'individus) constituerait donc une expérience inédite, pouvant conduire à un basculement des grilles de lecture des TIC. En mobilisant le concept d'emprise analytique développé par Claude Lemoine (2007), nous souhaitons appréhender les enjeux et les conséquences de la situation expérimentale que nous soumettons aux sujets. Parce qu'ils sont engagés dans une démarche de réflexion et de verbalisation de leurs propres pratiques, les individus interviewés s'approprieraient le fruit de nos échanges et, par là même, élaboreraient un travail cognitif d'acquisition de connaissances sur soi. La dimension diachronique de notre étude vise non seulement à observer l'évolution des usages numériques dans un intervalle temporel contrôlé, mais aussi à questionner la dimension de réflexivité émanant de notre méthodologie.

\section{Conception d'une méthodologie diachronique}

D'inspiration biographique, le protocole expérimental comprend deux entretiens individuels séparés d'une année d'intervalle (T0T1). Par l'entremise de l'interview compréhensive centrée reposant sur le vécu des sujets, le chercheur accompagne la verbalisation des pratiques communicationnelles pour en appréhender le sens.

Durant le premier entretien (T0), les cadres sont invités à produire le récit d'une journée-type de travail en se centrant sur les usages professionnels des TIC au gré des temporalités (privée, professionnelle, y compris l'enchevêtrement des deux sphères). En fin d'entrevue, la Technique des Incidents Critiques (Flanagan, $o p$. cit.) est amorcée de manière neutre pour questionner l'existence d'événements (positifs et/ou négatifs) qui seraient à l'origine de la construction de stratégies d'usage.

17 Voir Armengaud (2007). 
Le second entretien (qui nous intéresse plus précisément ici), réitéré un an après le premier, vise à appréhender de nouveau les usages quotidiens des TIC, dans le souci de caractériser les conditions d'apparition de nouvelles modalités attitudinales et/ou comportementales. Notre hypothèse diachronique consiste à tester la dimension de réflexivité introduite par notre démarche, autrement dit à appréhender un possible effet de conscientisation des pratiques entre T0 et T1. Puisque le premier entretien initie une activité réfléchissante ${ }^{18}$ sur les usages tels que relatés par les sujets euxmêmes, l'intérêt du second entretien vise à analyser autant le souvenir des échanges de T0 que la relation dialogique chercheur/sujet du point de vue de ce dernier. L'objectif de T1 est d'identifier, dans le témoignage des cadres, des éléments révélant l'existence d'une activité de distanciation.

Ainsi, au cours du méta-entretien ${ }^{19}$, les cadres de l'échantillon se dégagent d'une posture d' " objet d'étude » pour adopter celle d'analyste de leur propre vécu d'entretien. Après avoir introduit l'objet de la seconde rencontre (c'est-à-dire «faire le point sur l'année qui s'est écoulée ») et questionné les possibles changements personnels et professionnels, nous invitons le sujet à «raconter» l'entretien précédent. Le rôle du chercheur consiste à accompagner la verbalisation du souvenir de T0 et d'en extraire la substance la plus significative pour lui. Enfin, une place importante est accordée à l'expression des émotions ressenties lors du premier entretien, en prenant en compte le travail du temps ${ }^{20}$. L'entrevue se poursuit en abordant la perception de changement d'usage entre T0 et T1 ainsi qu'en interrogeant la survenue d'incidents critiques au cours de l'intervalle empirique.

La double collection d'entretiens a été soumise à une analyse par théorisation ancrée 21 dans le but d'élaborer une mise en sens

18 D'après Pierre Vermersch (1998), l'activité réfléchissante génère «une prise de conscience au sens basique de passage du vécu (de ce qui existe en acte) au représenté, permettant d'en prendre connaissance au plan des activités symboliques, représentatives, verbales, conceptuelles ».

19 Le fait de réaliser un entretien ayant pour objet un entretien précédent est appelé « méta-entretien » (Kridis, 2008).

20 Analyser un événement ne consiste pas seulement à appréhender l'instant de sa survenue, mais son expérience processuelle à travers ce qui lui précède et ce qui lui succède (Quéré, op. cit.).

21 Pour traiter la forte volumétrie du corpus (>1100 pages) et optimiser la 
des phénomènes, c'est-à-dire une compréhension des pratiques communicationnelles des cadres. Les parties suivantes de l'article présentent une partie des résultats de nos analyses qualitatives: celles relatives à la dimension longitudinale qui permettent une interprétation quant à la prise de position des sujets sur leur propre vécu d'entretien, et par là même à l'égard de son objet.

\section{Analyse du corpus longitudinal : les sujets à l'épreuve de la réflexivité}

Le dispositif méthodologique retenu pour cette étude et notamment son caractère longitudinal, qui a permis de réitérer des entretiens avec une même population de cadres 22 à un intervalle d'un an, autorise des observations intéressantes. Tout d'abord, la temporalité expérimentale permet de questionner l'évolution de la situation des sujets entre T0 et T1. Ensuite, la dimension diachronique présente l'opportunité d'appréhender les enjeux et les effets de la relation dialogique entre le chercheur et le sujet à partir du témoignage des cadres interviewés.

\section{L'édification d'un observatoire qualitatif des pratiques}

Échanger de nouveau avec les cadres rencontrés un an auparavant donne l'occasion de mettre en relation les possibles changements survenus dans leur vie personnelle et/ou professionnelle avec une modification des usages des TIC.

Nous observons ainsi, à travers le récit des sujets, qu'une augmentation de la responsabilité et de l'activité de l'entreprise ${ }^{23}$ s'accompagne généralement d'une extension de disponibilité et de travail en débordement. Certains cadres ${ }^{24}$ ont connu un changement de fonctions au cours de l'année. Par exemple, Christophe ${ }^{25}$ (cadre

manutention des données, l'analyse est informatisée à l'aide d'un logiciel CAQDAS (Computer-Aided Qualitative Data Analysis Software) : QSR Nvivo9.

22

L'échantillon de cadres rencontrés de nouveau lors du second entretien s'élève à 54 sujets. Le déficit de 8 sujets s'explique par des difficultés de rencontre (mobilité hors région Aquitaine, joignabilité impossible).

$2344,4 \%$ des sujets de l'échantillon sont concernés par cette situation.

243 sujets de l'échantillon.

25 Les verbatims cités en italique correspondent à des unités sémantiques issues de l'analyse de notre corpus. Afin de préserver l'anonymat et la confidentialité 
supérieur dans le secteur bancaire) qui assure moins d'activités managériales, constatait : "Beaucoup plus d'air dans les mails, c'est beaucoup plus confortable».

$\mathrm{Du}$ côté de la sphère privée, sept cadres de l'échantillon ont déclaré avoir vécu des changements entre T0 et T1. La naissance d'un enfant au cours de l'année a pu conduire les sujets concernés à reconfigurer leur connexion avec le travail en dehors des murs de l'entreprise. C'est le cas de Cécile, cadre intermédiaire dans le secteur RH, qui a choisi de rester joignable et d'assurer une veille informationnelle de l'activité professionnelle pendant son congé de maternité. Une situation de divorce 26 peut susciter un besoin de se déconnecter lors des temporalités privées pour mieux gérer la situation difficile. Durant une période de congé de maladie, les sujets concernés ${ }^{27}$ ont opté pour une connexion dans le but de s'assurer du bon déroulement des affaires en cours.

Au cours de l'année séparant les deux entretiens, onze cadres ont été concernés par un changement d'équipement technologique. La plupart sont désormais équipés de Smartphones par leur employeur. Les avantages du push-mail sont découverts, ainsi que l'inconvénient lié à l'extension de disponibilité.

À l'occasion de la seconde entrevue, nous avons questionné le rapport à la connexion des sujets au cours des périodes de vacances entre $\mathrm{T} 0$ et $\mathrm{T} 1$. La plupart des cadres qui déclaraient à $\mathrm{T} 0$ opter pour une connexion maîtrisée pendant les congés ${ }^{28}$, ont conservé leur stratégie. Onze sujets déclarent être moins connectés pendant leurs vacances que l'année précédente : ils ont notamment expérimenté la désactivation des applications push, opté pour la réhabilitation du différé dans la consultation du réseau, ou encore le filtrage des appels. Enfin, certains cadres ${ }^{29}$ se sont volontairement octroyé des congés de déconnexion absolue avec le travail, y compris certains néophytes.

des propos, tous les prénoms des sujets ont été changés.

26 Cela concerne 2 cadres de l'échantillon.

272 cadres de l'échantillon ont connu une période de congé de maladie entre $\mathrm{T} 0$ et $\mathrm{T} 1$.

28 Stratégie qui consiste à garder le contrôle sur les sollicitations professionnelles, à filtrer les appels, à gérer le flux informationnel pour éviter l'infobésité lors du retour de congés, et à attribuer des temps et des lieux précis pour les communications numériques (généralement négociés avec les proches et les collaborateurs).

29 Outre les sujets ne disposant pas de téléphone portable professionnel, cela concerne également des cadres qui en sont équipés et qui optent généralement pour 
Parce que la temporalité séparant les deux entretiens est relativement courte, le corpus d'événements marquants entre T0 et T1 apparaît comme restreint par rapport à celui recueilli lors des premiers échanges (questionnant l'épaisseur de l'histoire des sujets). Le récit de ces événements met en évidence la persistance des mésusages du mail (incidents négatifs) et l'opportunité de l'accès à distance au réseau professionnel (incidents positifs).

\section{Le vécu d'entretien biographique portant sur les usages numériques}

Dans sa fonction de méta-entretien, la seconde entrevue consiste en la réalisation d'un feedback portant sur T0. À partir du récit des sujets, nous cherchons à évaluer la propension du premier entretien biographique à se muer en terrain fécond de «mise en sens » de leurs propres pratiques.

Lorsqu'ils se remémorent $\mathrm{T} 0$, plus de la moitié des cadres ${ }^{30}$ en proposent un résumé en évoquant le thème de l'étude, l'objectif de la première rencontre, les étapes et la définition des rôles entre interviewé et interviewer. Généralement, cette description est empreinte de neutralité, mettant en exergue une forme de co-construction entre le sujet et le chercheur. D'autres cadres ont formulé une synthèse du premier entretien dans une perspective interne, racontant son contenu à la première personne du singulier (ce qui va dans le sens d'une réappropriation de l'étude). Ces récits donnent à penser l'expérience de $\mathrm{T} 0$ comme le résultat d'une interaction et nous renseignent quant à l'implication des sujets.

Pour la moitié la plus jeune de l'échantillon ${ }^{31}$, le premier entretien est décrit comme une opportunité de prise de recul, de "réflexion» sur leur propre rapport à la connexion. "On se met à distance », expliquait Astrid, cadre supérieur dans le secteur vitivinicole. T0 représenterait un événement biographique, "révélateur sur l'utilisation », pour cette partie de la population interviewée. Le travail discursif et réflexif qu'ils ont réalisé à T0 aurait eu un impact

une connexion maîtrisée pendant les vacances.

$30 \quad 57,4 \%$ des sujets de l'échantillon.

31 Cela représente $50 \%$ des cadres de l'échantillon : 6 cadres sur 7 âgés de moins de 34 ans ; 60,8\% des sujets âgés entre 35 et 44 ans. 
sur leur manière de voir et de se comporter avec les TIC : «ça m'a fait réaliser beaucoup de choses» (Rémi, cadre supérieur dans le secteur de la vente en ligne) ; "c'est-à-dire de les voir différemment » (Clément, cadre supérieur dans le secteur bancaire). Au cœur de leurs narrations, le champ lexical de l'image demeure fortement présent: «éclairer», "mettre en lumière », "photographier», ou encore «créer un horizon» constituent des exemples de termes employés par les sujets pour évoquer les effets du premier entretien. Les cadres composant la seconde moitié de l'échantillon ont, quant à eux, affirmé que l'expérience de T0 leur a permis de conforter leurs propres pratiques.

Étant donné que les usages professionnels des TIC ne sont pas objectivés au sein des organisations, nous avons questionné l'appréciation des sujets quant à l'aisance ou la difficulté que l'explicitation a impliqué lors du premier entretien. Pour $61,1 \%$ des cadres, cette tâche est perçue comme facile, eu égard à leur attitude clairvoyante à l'égard des dispositifs. Bien que peu mises en mots, les pratiques numériques seraient tout de même pensées par les sujets afin d'éviter les effets délétères des usages des TIC. D'autres cadres ont légitimé cette aisance d'explicitation par la conception du canevas d'entretien et l'accompagnement à la verbalisation. À l'inverse, une quinzaine de cadres considère cette tâche comme plutôt difficile du fait de disposer en majorité de connaissances procédurales en termes de TIC. Le souvenir d'un «effort » et d'un «temps d'adaptation » est présent dans leur récit.

Enfin, nous nous sommes intéressée à la portée pragmatique du premier entretien : la situation de communication de T0 est-elle vectrice de changement dans la relation des cadres aux TIC ? Les résultats vont dans le sens d'une rétroaction positive du premier entretien sur les pratiques communicationnelles des sujets. En effet, le vécu de T0 a suscité chez la majorité des cadres interviewés un renforcement de leurs stratégies: davantage d'autodiscipline, de filtrage, de conciliation entre les sphères de vie et de temps à soi.

Sur le court terme ${ }^{32}$, la manière de voir les dispositifs communicationnels a connu un bouleversement, comme le raconte George, cadre intermédiaire dans le secteur des transports pétroliers : «je réfléchissais plus quand j'allumais mon BlackBerry [...]. Je regardais aussi comment se comportaient les gens». La recherche 
d'un équilibre entre les domaines de vie fait partie des stratégies post-T0 les plus communes : cela consiste à réduire le travail en débordement pour préserver la qualité de relation avec leurs proches et mieux organiser leur temps de travail. «Le week-end, non, ça y est c'est fini. Basta! » (Boris, cadre supérieur dans le secteur des SSII). Les stratégies de filtrage sont également plus répandues qu'à T0 : les cadres optent pour une délégation de certains mails par un secrétariat, paramètrent leur messagerie pour trier les informations reçues, ou encore désactivent les technologies push. Étienne, cadre dirigeant dans le secteur de la production d'énergie, nous racontait : "maintenant, je construis des barrières humaines autour de moi [...]. Je délègue un peu plus, j'ai autour de moi des gens qui filtrent l'information». Enfin, les stratégies post-T0 prennent la forme de déconnexion dans des périodes où les sujets ont besoin de se ressourcer. Ils affirment la volonté de s'octroyer plus de temps pour eux-mêmes. Ces stratégies passent par l'investissement dans des activités sportives, associatives et sociales. "J'ai repris le sport, donc déjà quand on sort de la salle de sport on n'a pas tellement envie de ré-ouvrir l'ordinateur!» (Caroline, cadre dirigeante dans le secteur commercial).

\section{Conclusion}

Pour mieux comprendre le rapport que les cadres entretiennent à l'égard des TIC, l'entretien biographique, dans une approche longitudinale, se révèle être un protocole intéressant pour trois raisons principales. La première est d'ordre méthodologique dans une approche compréhensive. Reposant sur le vécu expérientiel des sujets, le corpus obtenu met en évidence le sens des pratiques numériques des cadres et de la mise en œuvre de stratégies d'ajustement. Le second argument de pertinence consiste en l'opportunité pour les sujets interviewés, en qualité de producteurs du récit, de se réapproprier leur parcours, et de recueillir le fruit de cet engagement réflexif de soi. Que la clairvoyance de leur rapport à la connexion ait été présente ou non avant $\mathrm{T} 0$, le premier entretien constitue pour les sujets une occasion de mieux comprendre et de donner du sens à leur usage professionnel des TIC. Enfin, ces résultats corroborent la perspective non-linéaire du parcours d'appropriation des dispositifs ${ }^{33}$ : les usagers peuvent voir et revoir leurs pratiques au gré des événements. 
Par ailleurs, la conduite de cette recherche a fait apparaître des aspects de médiation au sein du groupe social des cadres, catégorie de salariés souvent décrite comme silencieuse et en souffrance d'identité collective. En s'investissant dans cette recherche ${ }^{34}$, les sujets cherchent à confronter leurs pratiques à celles des autres, se « situer par rapport aux autres », et « apprendre des autres » à travers les résultats qui seront portés à leur connaissance. Le processus de réflexivité passerait ainsi d'un processus d'autoconnaissance à une auto-confrontation avec leurs pairs. Un sentiment d'isolement s'est dégagé de notre corpus : l'absence de prescription organisationnelle ${ }^{35}$ des usages numériques les arriment à l'autodiscipline, et l'absence d'espaces d'échanges formels sur cet objet ${ }^{36}$ freine les possibilités de régulation collective. En participant à l'étude, ils cherchent à satisfaire leur quête de lien social avec leurs pairs. À la lecture du manuscrit de thèse, la dynamique de réflexivité de leurs propres pratiques sera ainsi poursuivie, soit une étape supplémentaire qui s'inscrit au-delà de la temporalité contrôlée de l'étude.

34 Ces données émanent d'une catégorie de l'analyse relative aux attentes des sujets vis-à-vis de leur investissement dans une recherche universitaire portant sur leurs propres pratiques des TIC. Ce questionnement vient clôturer le dernier entretien (T1) mené individuellement auprès des cadres.

35 Les résultats de la présente étude mettent en évidence que l'organisation du travail «ne pense pas » les usages numériques. L'enquête menée auprès de professionnels de la santé au travail et de partenaires sociaux (Felio, 2014) confirme l'absence de grille de lecture des pratiques communicationnelles qui entrave l'appréhension du réel de l'activité, tout comme le processus d'alerte sur la dégradation des conditions de travail. Par ailleurs, le terrain de recherche portant sur les DRH (Boudokhane-Lima et Carayol, op. cit.) montre la posture ambiguë des directions d'entreprise face à ce phénomène, relayant la responsabilité des problématiques d'usage aux individus (les cadres) tout en reconnaissant les risques médiatisés par les TIC dans la sphère professionnelle.

36 Si des espaces institutionnalisés par l'organisation ne sont pas construits, l'analyse de notre corpus fait tout de même apparaitre l'existence d'une forme de «plainte rituelle» (Foli, 2008) entretenue par les cadres sur la question des TIC au travail. Se plaindre des sollicitations permanentes et de la surcharge des boîtes mail serait très fréquent dans les interactions entre cadres : il s'agit cependant d'échanges superficiels qui ne revêtent pas de caractère sérieux et ne permettent pas de libérer une parole sur les contraintes, les sources des effets pathiques et les stratégies individuellement mobilisées. Ce discours de surface officie un rôle de préservation des relations sociales : il permet de restaurer le sentiment de «faire collectif» et d'attester son sentiment d'appartenance au groupe social des cadres. 
Somme toute, il nous semble important de formuler quelques réserves quant à certaines implications que les résultats de l'étude présentés ici pourraient induire concernant notre approche méthodologique. En particulier, ces résultats pourraient donner à penser que la méthodologie déployée favoriserait, du côté des acteurs interviewés, l'émergence d'une posture critique favorable à des formes d'émancipation vis-à-vis des TIC, voire pourrait être bénéfique à la constitution d'un espace de concertation entre les cadres pour une définition collective des formes de régulation sociales intégrant les TIC. Or, d'une part, certains éléments du corpus d'entretiens peuvent conduire à nuancer la portée strictement émancipatrice des pratiques. En effet, des conséquences sur les dimensions de pouvoir des cadres-encadrants peuvent émaner de l'investissement dans la recherche en tant que sujets d'étude : lors du second entretien, les managers de l'échantillon semblent se responsabiliser davantage face aux usages numériques de leurs équipes et déclarent développer des formes de régulations en vue d'une normalisation des pratiques ${ }^{37}$. D'autre part, les fonctions de médiation sociale allouées au design de la recherche ne semblent pas exclusivement orientées dans le sens d'une rétroaction positive entre les membres d'un même groupe social. De fait, le discours des cadres est souvent caractérisé par des formes de distanciation vis-à-vis de leurs pairs pour valoriser leurs propres arts de faire, pour se différencier de ceux qui subissent les effets délétères des usages techniques et qui contribuent à perpétrer les problématiques en lien avec les TIC $^{38}$. La participation à l'étude pourrait également se faire une place dans le jeu de la concurrence et de la compétition entre les cadres: quelques sujets évoquent notamment le fait de "beaucoup parler de cet entretien autour de [soi] ».

CINDY FELIO ${ }^{39}$

Laboratoire MICA (EA 4426) Université Bordeaux Montaigne

37 Ces tentatives de régulations portent majoritairement sur la gestion du mail, média le plus décrié pour les problèmes de communication que son usage occasionne. 38 L'impression d'être un « zombie », d'adopter une posture «marginale » par rapport aux autres, en affirmant entretenir une relation de maitrise avec les TIC.

39 Lauréate du $2^{\mathrm{e}}$ prix « jeunes chercheurs » 2014 de la SFSIC. 


\section{BIBLIOGRAPHIE}

Amado G., (2010), Subjectivité limitée, travail et résonance psychique, in Clot Y. et Lhuilier D. (dirs.), Travail et santé, ouvertures cliniques, Toulouse, Érès, pp. 65-77.

Armengaud F., (2007), La pragmatique, Paris, PUF, 127 p.

Barbot M.-J. et Lancien T., (2003), Médiation, médiatisation et apprentissages, Lyon, ENS Éditions, 152 p.

Bensa A. et Fassin É., (2002), Les sciences sociales face à l'événement, in Terrain, $\mathrm{n}^{\circ} 38$, pp. 5-20.

Bernard F., (2000), Le lien communicationnel en organisation, in Sciences de la Société, $\mathrm{n}^{\circ}$ 50/51, pp. 25-45.

Bobillier-Chaumon M.-É., (2012), L'impact des technologies de communication sur les cadres. Rapport de recherche GRePS / APEC, http://recherche.univ- lyon2.fr/ greps/IMG/pdf/Rapport_GREPS_012012-V-Finale.pdf, consulté le 01/09/13.

Boudokhane-Lima F. et Carayol V., (2014), Les Directeurs des Ressources Humaines face au phénomène d'hyperconnexion des cadres, Colloque International « Déconnexions Volontaires aux TIC », Université de Pau et des Pays de l'Adour.

Bouillon J.-L., (2003), Pour une approche communicationnelle des processus de rationalisation cognitive des organisations : contours, enjeux et perspectives, $\mathrm{X}^{\mathrm{e}}$ Colloque bilatéral franco-roumain, CIFSIC Université de Bucarest, http:// archivesic.ccsd.cnrs.fr/docs/00/06/23/71/PDF/sic_00000949.pdf, consulté le 26/07/14.

Bouilloud J.-P., (2012), Entre l'enclume et le marteau. Les cadres pris au piège, Paris, Éditions du Seuil, 222 p.

Broadbent S., (2012), Le temps réel et la communication personnelle, in Lejeune Y. (dir.), TIC 2013, les nouveaux temps réels, Limoges, FYP Éditions, pp. 11-12.

Brousseau É. et Rallet A., (1997), Le rôle des technologies de l'information et de la communication dans les changements organisationnels, in Guilhon B., Huard P., Orillard M. et al. (dirs.), Économie de la connaissance et Organisation ; Entreprises, territoires, réseaux, Paris, L'Harmattan, pp. 286-309.

Brynjolfsson E. et Hitt L. M., (2000), Beyond Computation: Information Technology, Organizational Transformation and Business Performance, in Journal of Economic Perspectives, vol. 14, n 4, pp. 23-48.

Burnier M., (2012a), Regards sur les conditions de travail et les TIC : entretien avec Yves Lasfargue, in Terminal, $\mathrm{n}^{\circ} 110$, pp. 77-89.

Burnier M., (2012b), Regards sur le travail et les TIC. Entretien avec Jean-Pierre Durand, in Terminal, $\mathrm{n}^{\circ} 110$, pp. 69-76.

Carayol V., (2005), Contre l'urgence, la paresse, l'inaction, la lenteur, in Carayol V. (dir.), Vivre l'urgence dans les organisations, Paris, L'Harmattan, pp. 65-81.

Cherba M. et Vásquez C., (2012), Communication organisationnelle et santé : état de l'art et défis, $80^{\mathrm{e}}$ congrès de l'ACFAS, Montréal, Québec, http://www.grms.uqam. ca/upload/files/Actes_de_colloque/ACTE_4_PDF.pdf, consulté le 03/05/13.

Chevallet R. et Moatty F., (2012), Impacts des TIC sur les rythmes, l'autonomie et le contrôle du travail, in Klein T. et Ratier D. (dirs.), L'impact des TIC sur les 
conditions de travail. Direction Générale du Travail, Rapport \& Documents, $\mathrm{n}^{\circ}$ 49, pp. 83-103.

Cochoy F. et Terssac (de) G., (2000), Au-delà de la traçabilité : la mappabilité. Deux notions connexes mais distinctes pour penser les normes de management, in Serverin E. et Berthoud A. (dirs.), La production des normes entre État et société civile, Paris, L'Harmattan, pp. 239-249.

Cordelier B., Vasquez C. et Mahy I., (2011), L'organisation en mouvement : action, temporalité et processus, in Revue internationale de communication sociale et publique, $\mathrm{n}^{\circ} 5$, I-VIII.

Datchary C. et Licoppe C., (2007), La multi-activité et ses appuis : l'exemple de la "présence obstinée »des messages dans l'environnement de travail, in @ctivités, $\mathrm{n}^{\circ}$ 4, pp. 4-29.

Dejours C., (1988), Plaisir et souffrance dans le travail. Tome 2, Orsay, Éditions de l'AOCIP, 269 p.

De La Broise P., (2008), L'entretien biographique: plus qu'une méthode, une méthodologie pour la recherche en communication organisationnelle, in Bouzon A. et Meyer V. (dirs.), La Communication des organisations : entre recherche et action, Paris, L'Harmattan, pp. 105-116.

Felio C., (2014), L'enjeu psychosocial de l'usage des TIC: témoignages d'intervenants en santé au travail, in Lagabrielle Christine et Laberon Sonia (dirs.), Santé au travail et risques psychosociaux : tous préventeurs ?, Paris, L'Harmattan, à paraître.

Felio C., (2013), Pratiques communicationnelles des cadres. Usage intensif des TIC et enjeux psychosociaux, Thèse de Doctorat en Sciences de l'information et de la communication, Université Bordeaux Montaigne, $486 \mathrm{p}$.

Felio C. et Carayol V., (2013), Apports de la technique des incidents critiques à l'étude des pratiques d'hyper connexion des cadres, in Vacher Béatrice, Le Moënne Christian et Kiyindou Alain (dirs.), Communication et débat public: les réseaux numérique au service de la démocratie ?, Paris, L'Harmattan, pp. 229-238.

Flanagan J. C., (1954), The Critical Incident Technique, in Psychological Bulletin, vol. $51, n^{\circ} 4$, pp. 327-359.

Foli O., (2008), Plaintes, normes et intégration. Le cas d'une organisation bureaucratique, Thèse de Doctorat en Sociologie, Université Paris Dauphine, 429 p.

Gonzàlez V. M. and Mark G., (2004), "Constant, constant, multi-tasking craziness »: managing multiple working spheres, SIGCHI Conference on Human Factors in Computing Systems, New York, USA, 2004, pp. 113-120.

Jeanneret Y., (2009), La relation entre médiation et usage dans les recherches en information-communication en France, in RECIIS, vol. $3, \mathrm{n}^{\circ} 3$.

Kalika M. et Romelaer P., (2006), Recherches en management et organisations, Paris, Economica, 2006, 663 p.

Kridis N., (2008), Communication et Innovation. Champs, méthodes, interventions, Paris, L'Harmattan, 160 p.

Lafranchise N., (2013), Restaurer les liens sociaux et l'espace communicationnel par les groupes de codéveloppement professionnel, III' Journée d'Études de l'ORRPSA, Université de Bordeaux.

Leclerc-Olive M., (1997), Le dire de l'événement, Villeneuve-d'Ascq, Presses Universitaires du Septentrion, $260 \mathrm{p}$. 
Le Goff J.-P., (1995), Le mythe de l'entreprise, Paris, La Découverte, 307 p.

Lemoine C., (2007), De la question à l'auto-questionnement : questionner, source d'emprise et d'appropriation multiple, vol. 13, n 4, pp. 5-24.

Lépine V., (2001), Dispositifs de groupware : entre prescriptions et usages réels, Communication \& Organisation, $\mathrm{n}^{\circ} 19$, pp. 39-54.

Menga D., (2012), Traitement de données et temps réel, in Lejeune Y. (dir.), op. cit., pp. 138-145.

Molinier P. et Flottes A., (2012), Travail et santé mentale : approches cliniques, in Travail et Emploi, $\mathrm{n}^{\circ} 129$, pp. 51-66.

Mucchielli A., (2009), Dictionnaire des méthodes qualitatives en sciences humaines, Paris, Armand Colin, 303 p.

Quéré L., (1990), Agir dans l'espace public, in Pharo P. et Quére L. (dirs.), Les formes de l'action, Paris, EHESS, pp. 85-112.

Quére L., (2006), Entre fait et sens, la dualité de l'évènement, in Réseaux, vol. 139, $\mathrm{n}^{\circ}$ 5, pp. 183-218.

Riccio P.-M. et Commandre M., (2009), Une approche systémique et non-linéaire de l'émergence d'une communauté scientifique, Journées d'Études Dynamique de Réseaux, Information, Complexité et Non-Linéarité «TIC'IS 2009 », Marseille, http://hal.archives-ouvertes.fr/docs/00/81/24/35/PDF/_44.pdf, consulté le 29/04/13.

Rosa H., (2010), Accélération : Une critique sociale du temps, Paris, La Découverte, $474 \mathrm{p}$.

Sarnin P., (2012), L'activité des cadres passée au crible, in Personnel, Revue de $l^{\prime}$ ANDRH, n ${ }^{\circ} 531$, pp. 52-54.

Vannereau J., (2012), L'invention managériale : une compétence cardinale pour les situations organisationnelles complexes, in Revue de Psychologie du Travail et des Organisations, $\mathrm{n}^{\circ} 17$, pp. 186-207.

Vermersch P., (1998), Détacher l'explicitation de la technique d'entretien?, in Expliciter, $\mathrm{n}^{\circ} 25$, pp. 1-15.

Walkowiak E. et Greenan N., (2005), Informatique, organisation du travail et interactions sociales, in Economie et statistique, $\mathrm{n}^{\circ} 387$, pp. 35-63. 